PROCEEDINGS OF THE

AMERICAN MATHEMATICAL SOCIETY

Volume 132, Number 10, Pages 2911-2915

S 0002-9939(04)07492-1

Article electronically published on May 12, 2004

\title{
NORM-CLOSURE OF THE BARRIER CONE IN NORMED LINEAR SPACES
}

\author{
SAMIR ADLY, EMIL ERNST, AND MICHEL THÉRA
}

(Communicated by Jonathan M. Borwein)

\begin{abstract}
The aim of this note is to characterize the norm-closure of the barrier cone of a closed convex set in an arbitrary normed linear space by means of a new geometric object, the temperate cone.
\end{abstract}

\section{INTRODUCTION AND NOTATION}

Throughout the paper, we assume that $X$ is a real normed linear space with continuous dual $X^{*}$ and we denote by $\|\cdot\|$ and $\|\cdot\|_{*}$ the norms on $X$ and $X^{*}$, respectively. Let $C$ be a convex subset of $X$, and let

$$
\mathcal{B}(C)=\left\{f \in X^{*}: \sup _{x \in C}\langle f, x\rangle<\infty\right\}
$$

denote the barrier cone to $C$. As usual (see Rockafellar [4 as a reference book for the finite-dimensional case), we use the notation $C^{\infty}$ for the recession cone of the closed convex set $C$, that is,

$$
C^{\infty}=\left\{v \in X: \quad \forall \lambda>0, x_{0} \in C, x_{0}+\lambda v \in C\right\} .
$$

Denoting by $\langle\cdot, \cdot\rangle$ the duality pairing between $X^{*}$ and $X$, we recall that the negative polar cone $C^{\circ}$ of a closed convex set $C$ is

$$
C^{\circ}=\left\{f \in X^{*}:\langle f, w\rangle \leq 0 \quad \forall w \in C\right\} .
$$

The main objective of this note is to give a direct characterization of the normclosure of the barrier cone for a closed convex set in a general normed linear space. It is well known (see for instance [6], Ex. 2.45) that the weak ${ }^{*}$-closure of the barrier cone of $C$ is the polar of the recession cone of $C$,

$$
\text { weak }^{*}-\operatorname{cl}(\mathcal{B}(C))=\left(C^{\infty}\right)^{\circ} .
$$

When the underlying linear space $X$ is reflexive, since the barrier cone of $C$ is convex, its weak $k^{*}$-closure coincides with its norm-closure, and thus the norm-closure $\overline{\mathcal{B}(C)}$ of $\mathcal{B}(C)$ is characterized in $X^{*}$ by the formula:

$$
\overline{\mathcal{B}(C)}=\left(C^{\infty}\right)^{\circ} \text {. }
$$

Received by the editors July 15, 2002.

2000 Mathematics Subject Classification. Primary 46N10, 47N10.

Key words and phrases. Fenchel conjugate, barrier cone, temperate cone, linearly bounded set, recession cone.

The third author's research was partially supported by the French Chilean Scientific Cooperation Programme ECOS under grant C00E05 and by NATO Collaborative Linkage Grant 978488. 
When $X$ is not a reflexive Banach space, the weak ${ }^{*}$-closure of $\mathcal{B}(C)$ may significantly be larger than its norm-closure, and thus relation (1.1) may fail. This situation occurs for instance when $X=L^{1}(0,1)$ and $C=\left\{f \in L^{1}(0,1): 0 \leq\right.$ $\left.f(x) \leq \frac{1}{x}\right\}$. Then, obviously, $C$ is a closed convex set and $C^{\infty}=\{0\}$. The sets $K=\left\{f \stackrel{x}{\in} X^{*}=L^{\infty}(0,1): 1 \leq f\right\}$ and $\mathcal{B}(C)$ are disjoint; since the interior of $K$ is non-void, it follows that $\overline{\mathcal{B}(C)} \neq X^{*}=\left(C^{\infty}\right)^{\circ}$.

In order to characterize the norm-closure of the barrier cone in a general normed linear space, we introduce and study a new object that we call the temperate cone of a closed convex set. The main result, Theorem [2.1] states that the temperate cone of a closed convex set $C$ is the norm-closure of the barrier cone of $C$ for every closed and convex subset $C$ of a normed linear space.

This result leads to the description (Proposition 2.5) of the class of all closed and convex sets satisfying relation (1.1) in a general normed linear space.

In the sequel, the symbol $\mathbb{B}_{X}$ is used to denote the closed unit ball in $X$, and a closed convex set is called linearly bounded if $C^{\infty}=\{0\}$.

\section{The NORM-ClOSURE OF THE BARRIER CONE}

Let $C$ be a closed convex set in a normed linear space $X$. Along with the barrier cone $\mathcal{B}(C)$ of the set $C$, we define the temperate cone of $C$ :

$$
\mathcal{T}(C)=\left\{f \in X^{*}: \lim _{r \rightarrow \infty}\left(\inf _{x \in C,\langle f, x\rangle \geq r} \frac{\|x\|}{r}\right)=\infty\right\} .
$$

Since $\inf _{x \in \emptyset}\|x\|=\infty$, it follows that $\mathcal{B}(C) \subseteq \mathcal{T}(C)$.

The following theorem states the main result of this note.

Theorem 2.1. For every closed convex set $C$ of a normed linear space $X$ the temperate cone is the norm-closure of the barrier cone.

Let $C$ be a given closed convex set. We recall that the indicator function of $C$ is the functional $\iota_{C}$ defined by

$$
\iota_{C}(x)= \begin{cases}0 & \text { if } x \in C, \\ \infty & \text { if } x \in X \backslash C .\end{cases}
$$

Given an extended real-valued function $\Psi: X \rightarrow \mathbb{R} \cup\{+\infty\}$, recall that the Fenchel conjugate of $\Psi$ is the function $\Psi^{*}: X^{*} \rightarrow \mathbb{R} \cup\{+\infty\}$ given by

$$
\Psi^{*}(f):=\sup _{x \in X}\{\langle f, x\rangle-\Psi(x)\} .
$$

The barrier cone of a closed convex set $C$ coincides with the effective domain of the Fenchel conjugate of its indicator function, i.e., the set of all the points where this Fenchel conjugate is finite. Since, obviously, the epigraph of $\iota_{C}$ denoted by epi $\iota_{C}$ coincides with $C \times[0,+\infty)$, we have $\mathcal{B}\left(\right.$ epi $\left.\iota_{C}\right)=\mathcal{B}(C) \times(-\infty, 0]$ and therefore,

$$
\mathcal{B}\left(\text { epi } \iota_{C}\right) \cap\left(X^{*} \times\{-1\}\right)=\mathcal{B}(C) \times\{-1\} \text {. }
$$

Obviously, the domain of $\Psi^{*}$ denoted by dom $\Psi^{*}$ is connected to the barrier cone of the epigraph of $\Psi$ through the following equivalence:

$$
g \in \operatorname{dom} \Psi^{*} \Leftrightarrow(g,-1) \in \mathcal{B}(\text { epi } \Psi) .
$$


This yields that dom $\Psi^{*} \times\{-1\}$ is the intersection of the barrier cone of epi $\Psi$ with the hyperplane $X^{*} \times\{-1\}$ of $X^{*} \times \mathbb{R}$ :

$$
\operatorname{dom} \Psi^{*} \times\{-1\}=\mathcal{B}(\text { epi } \Psi) \cap\left(X^{*} \times\{-1\}\right) .
$$

Finally, from relations (2.2) and (2.3) we deduce that

$$
\mathcal{B}(C)=\operatorname{dom} \iota_{C}^{*} \text {. }
$$

Let us recall the following well-known characterization of the norm-closure of the domain of the Fenchel conjugate of an extended real-valued, proper, convex and lower semicontinuous functional.

Theorem 2.2. Let $\Psi: X \mapsto \mathbb{R} \cup\{+\infty\}$ be an extended real-valued proper convex and lower semicontinuous functional. Then $0 \in \overline{\operatorname{dom}\left(\Psi^{*}\right)}$ if and only if the map $x \mapsto(\Psi(x)+\varepsilon\|x\|)$ is bounded from below for every $\varepsilon>0$.

Remark 2.3. For the reflexive case, Theorem 2.2 goes back (in a slightly different form) to [5]. The non-reflexive setting has been considered for the first time in [3] (the reader is invited to consult Example 25 in [6], where a clear, detailed and self-contained analysis is given).

From Theorem 2.2 we deduce that a given element $f$ of $X^{*}$ belongs to the normclosure of $\mathcal{B}(C)$ if and only if the map $x \mapsto \iota_{C}(x)-\langle f, x\rangle+\varepsilon\|x\|$ is bounded from below for every $\varepsilon>0$.

Theorem 2.1 will thus be verified by proving that the above property characterizes the elements of the temperate cone of the closed convex set $C$.

For every $f \in X^{*}$, let us use the notation $C_{f}$ for $\{x \in X:\langle f, x\rangle \geq\|x\|\}$. The proof of Theorem 2.1 relies on the following characterization of the temperate cone.

Lemma 2.4. Let $C$ be a closed convex set in a normed linear space $X$. A continuous linear functional $f \in X^{*}$ belongs to the temperate cone $\mathcal{T}(C)$ if and only if the set $C \cap C_{\lambda f}$ is bounded for every $\lambda>0$.

Proof of Lemma 2.4 Let us first prove that for every $f \in \mathcal{T}(C)$ and $\lambda>0$, the set $C \cap C_{\lambda f}$ is bounded. From relation (2.1) it follows that there is $\bar{r}>0$ such that

$$
\inf _{x \in C,\langle f, x\rangle \geq r} \frac{\|x\|}{r} \geq(1+\lambda) \quad \forall r \geq \bar{r} .
$$

Let $x \in C$ be such that $\langle f, x\rangle \geq \bar{r}$. Applying relation (2.4) to $r=\langle f, x\rangle$ yields

$$
\|x\| \geq(1+\lambda)\langle f, x\rangle>\langle\lambda f, x\rangle \quad \forall x \in C,\langle f, x\rangle \geq \bar{r} .
$$

Let $x \in C \cap C_{\lambda f}$, which means that $x \in C$ and $\|x\| \leq\langle\lambda f, x\rangle$. From relation (2.5) it follows that $\langle f, x\rangle \leq \bar{r}$. Accordingly, $\|x\| \leq \lambda \bar{r}$, and so $C \cap C_{\lambda f} \subseteq \lambda \bar{r} \mathbb{B}_{X}$.

In order to complete Lemma 2.4 it suffices to prove that $f \in \mathcal{T}(C)$ provided that the set $C \cap C_{\lambda f}$ is bounded for every $\lambda>0$. Pick $M>0$. Since the set $C \cap C_{M f}$ is bounded, there is $\bar{r}>0$ such that $\left(C \cap C_{M f}\right) \subseteq \bar{r} \mathbb{B}_{X}$. Accordingly, for every $x \in C \cap C_{M f}$ it follows that $\|x\| \leq \bar{r}$; since $\langle f, x\rangle \leq\|f\|_{*}\|x\|$ for every $x$ from $X$, we deduce that $\langle f, x\rangle \leq\|f\|_{*} \bar{r}$ for every $x \in C \cap C_{M f}$. Thus, for every $x \in C$ such that $\langle f, x\rangle>\|f\|_{*} \bar{r}$ we have $x \notin C_{M f}$; that is, $\|x\|>M\langle f, x\rangle$. Consequently, for every $r>\|f\|_{*} \bar{r}$ and $x \in C$ such that $\langle f, x\rangle \geq r$ we have

$$
\frac{\|x\|}{r}>\frac{M\langle f, x\rangle}{r} \geq M .
$$


Hence,

$$
\inf _{x \in C,\langle f, x\rangle \geq r} \frac{\|x\|}{r} \geq M \quad \forall r>\|f\|_{*} \bar{r},
$$

and the conclusion of Lemma 2.4 follows.

Proof of Theorem 2.1] Remark that the map $x \mapsto \iota_{C}(x)-\langle f, x\rangle+\varepsilon\|x\|$ is bounded from below if and only if

$$
\exists \mu_{\epsilon} \in \mathbb{R} \text { such that }\langle f, x\rangle \leq \varepsilon\|x\|+\mu_{\varepsilon}, \quad \forall x \in C .
$$

In view of Lemma 2.4 we have only to prove that relation (2.6) holds for every $\varepsilon>0$ if and only if $C \cap C_{\lambda f}$ is bounded for every $\lambda>0$.

Assume that for some $f \in X^{*}$ relation (2.6) holds for every $\varepsilon>0$, and fix $\lambda>0$. Taking $\varepsilon=\frac{1}{2 \lambda}$ in relation (2.6) yields

$$
\exists \bar{\mu} \in \mathbb{R} \text { such that }\langle f, x\rangle \leq \frac{1}{2 \lambda}\|x\|+\bar{\mu}, \quad \forall x \in C .
$$

According to the definition of $C_{\lambda f}$, for every $x \in C \cap C_{\lambda f}$ we have

$$
\|x\| \leq\langle\lambda f, x\rangle .
$$

From the two previous relations we deduce

$$
\|x\| \leq \frac{1}{2}\|x\|+\lambda \bar{\mu} \forall x \in C \cap C_{\lambda f}
$$

and consequently we have $C \cap C_{\lambda f} \subseteq 2 \lambda \bar{\mu} \mathbb{B}_{X}$.

Thus, if the map $x \mapsto \iota_{C}(x)-\langle f, x\rangle+\varepsilon\|x\|$ is bounded from below for every $\varepsilon>0$, then $C \cap C_{\lambda f}$ is bounded for every $\lambda>0$.

Conversely, assume that $C \cap C_{\lambda f}$ is bounded for every $\lambda>0$, and fix $\varepsilon>0$. The set $C \cap C_{f / \varepsilon}$ being bounded, there exists $\gamma>0$ such that $\|x\| \leq \gamma$ for every $x \in C \cap C_{f / \varepsilon}$.

Let $x \in C \backslash C_{f / \varepsilon}$; accordingly,

$$
\left\langle\frac{f}{\varepsilon}, x\right\rangle \leq\|x\|
$$

On the other hand, for every $x \in C \cap C_{f / \varepsilon}$ it follows that

$$
\left\langle\frac{f}{\varepsilon}, x\right\rangle \leq \frac{\|f\|_{*}}{\varepsilon}\|x\| \leq \frac{\|f\|_{*}}{\varepsilon} \gamma .
$$

From relations (2.7) and (2.8) it follows that

$$
\langle f, x\rangle \leq \varepsilon\|x\|+\tilde{\mu} \quad \forall x \in C,
$$

where $\tilde{\mu}=\gamma\|f\|_{*}$. Thus, the map $x \mapsto \iota_{C}(x)-\langle f, x\rangle+\varepsilon\|x\|$ is bounded from below for every $\varepsilon>0$, and the proof of Theorem 2.1 is completed.

From Theorem 2.1 and Lemma 2.4 we obtain the following characterization of the family of all closed convex sets fulfilling condition (1.1).

Proposition 2.5. For every closed convex set $C$ of a normed linear space $X$, the following two facts are equivalent:

i) $\overline{\mathcal{B}(C)}=\left(C^{\infty}\right)^{\circ}$;

ii) $C \cap C_{f}$ is bounded for every $f \in\left(C^{\infty}\right)^{\circ}$. 
Remark 2.6. When $C$ is linearly bounded, Proposition 2.5 states that $\overline{\mathcal{B}(C)}=X^{*}$ if and only if $C \cap C_{f}$ is bounded for every $f \in X^{*}$. Condition $i i$ ) from Proposition 2.5. defines the family of conically bounded sets, which is a subclass of the class of linearly bounded sets for which holds the property of density of the barrier cone in $X^{*}$.

\section{ACKNOWLEDGMENTS}

The authors gratefully acknowledge a referee for a careful reading of the manuscript and for useful suggestions concerning the proof of Theorem 2.1.

\section{REFERENCES}

[1] J.-P. Aubin, Optima and Equilibria, An Introduction to Nonlinear Analysis, Graduate Texts in Mathematics, vol. 140, Springer-Verlag, Berlin, 1993. MR 94b:49002

[2] D. Azé, Éléments d'Analyse Convexe et Variationnelle, Ellipse, 1997.

[3] J. M. Borwein and J. D. Vanderwerff, Convergence of Lipschitz Regularizations of Convex Functions, J. Funct. Anal. 128, 139-162, 1995. MR 96m:49022

[4] R. T. Rockafellar, Convex Analysis, Princeton University Press, Princeton, NJ, 1968. MR 43:445

[5] R. T. Rockafellar, Level Sets and Continuity of Conjugate Convex Functions, Trans. Amer. Math. Soc. 123, 46-63, 1966. MR 33:544

[6] C. Zălinescu, Convex Analysis in General Vector Spaces, World Scientific, River Edge, NJ, 2002. MR 2003k:49003

LACO, Université de Limoges, 123 Avenue A. Thomas, 87060 Limoges Cedex, France E-mail address: adly@unilim.fr

Laboratoire de Modélisation en Mécanique et Thermodynamique (LMmT), Casse 322, Faculté de Sciences et Techniques de Saint Jérome, Avenue Escadrille NormandieNiemen 13397 Marseille Cedex 20, France

E-mail address: Emil.Ernst@univ.u-3mrs.fr

LACO, Université de Limoges, 123 Avenue A. Thomas, 87060 Limoges Cedex, France

E-mail address: michel.thera@unilim.fr 